\title{
Symptoms of Depression and Anxiety Among Black Medical Students: the Role of Peer Connectedness and Perceived Discrimination
}

\author{
Adam J. Milam ${ }^{1,2} \oplus \cdot$ Osose Oboh ${ }^{3} \cdot$ Zackary Brown $^{4} \cdot$ Jennifer Edwards-Johnson ${ }^{5} \cdot$ Aliyya Terry $^{6} \cdot$ Clara B. Barajas $^{7}$. \\ Kevin M. Simon ${ }^{8,9} \cdot$ C. Debra M. Furr-Holden ${ }^{1,5,7,10}$
}

Received: 1 May 2021 / Revised: 31 August 2021 / Accepted: 20 September 2021 / Published online: 1 October 2021

(c) W. Montague Cobb-NMA Health Institute 2021

\begin{abstract}
This study examined the association between discrimination, peer connectedness, and mental health symptoms among Black medical students. Data were collected from a convenience sample of Black medical students via an anonymous electronic questionnaire $(n=733)$ in year 2020. The Patient-Reported Outcomes Measurement Information System Depression and Anxiety forms were used to measure depression and anxiety symptoms. Structural equation modeling was used to examine the association between discrimination, peer connectedness, and mental health symptoms (Mplus 7.3). The majority of the participants were female (80\%), approximately $40 \%$ were third or fourth year medical school students, and $13 \%$ had a clinical diagnosis of depression/anxiety before medical school. About half of the students reported being watched more closely than their classmates, and $66 \%$ reported feeling the need to work twice as hard as others to get the same treatment or evaluation. The majority of students reported that their peers were supportive of their academic success $(60.7 \%)$, and 53\% reported that students often or always invited them to social outings. The mean T-score for depressive symptoms was $53.6(\mathrm{SD}=7.8)$, and the mean T-score for anxiety symptoms was $58.6(\mathrm{SD}=8.4)$. Overall, findings indicated a high prevalence of anxiety and depression symptoms among Black medical students, and increased discrimination was associated with more mental health symptoms among males. Additionally, increased peer connectedness was associated with fewer symptoms of anxiety among males and females and fewer depressive symptoms among females. Addressing discrimination among medical students may improve mental health among Black medical students.
\end{abstract}

Keywords Discrimination · Depression · Anxiety · Black · African American

Adam J. Milam

milam.adam@mayo.edu

1 Johns Hopkins Bloomberg School of Public Health, Baltimore 21205, USA

2 Mayo Clinic Arizona, Phoenix, USA

3 College of Human Medicine, Michigan State University, Flint, MI, USA

4 Howard University School of Medicine, Washington, DC, USA

5 Department of Family Medicine, Michigan State University, Flint, MI, USA

6 Kaiser Permanente, Oakland, CA, USA

7 College of Human Medicine Division of Public Health, Michigan State University, Flint, MI, USA

8 Boston Children's Hospital, Boston, MA, USA

9 Harvard Medical School, Boston, MA, USA

10 Department of Epidemiology and Biostatistics, Michigan State University, Flint, MI, USA
Medical students and physicians in the USA report higher rates of psychological distress, depressive symptoms, suicidal ideation, and attempted suicide compared to the general population [1-6]. A meta-analysis from 2016 found that the prevalence of depression or depressive symptoms among medical students in the USA was $26.7 \%$ ( $n=42$ studies) [6]. The same meta-analysis found that only $15.7 \%$ of medical students who were screened positive for depression sought psychiatric care [6]. These symptoms appear to have a disparate prevalence among demographic groups, with studies showing that Black medical students are more susceptible to symptoms of depression and anxiety and more likely to report moderate to severe depression and anxiety symptoms than their White counterparts [7]. Black medical students who reported experiences of discrimination were also more likely to experience symptoms of depression and anxiety. Studies examining gender also reveal disparities; female medical students are more likely to have symptoms of 
depression and anxiety, mirroring gender differences in the prevalence of depression and anxiety in the general population $[7,8]$.

Several factors contribute to the development of mental health problems [1,9-11]. Common risk factors for depression and depressive symptoms include major life changes, trauma or stress, personal or family history of depression, and physical illness and medications [1,9]. Risk factors for anxiety include a family history of anxiety or mental illness, exposure to stressful and negative life events, and physical health challenges [1,9-11]. Medical education and training involve many of the aforementioned risk factors related to poor mental health and others, such as role transition, decreased sleep, relocation, and increased feelings of isolation $[1-3,9,12]$. Additional stressors include financial burdens, exposure to patient's suffering and death, workload, and overall academic pressure that may also contribute to students' mental health [1, 7]. Further, burnout rates (mental exhaustion due to prolonged stress) are higher among physicians and medical trainees than the general population $[3,12,13]$. Discrimination as a stressor can be harmful to health, especially when discriminatory experiences are considered uncontrollable and unexpected [14]. Even perceived discrimination can lead to stress overload. The Institute of Medicine's report on unequal treatment documented an association between perceived discrimination and poorer health status, including mental health [15]. While researchers have suggested that race-related stress on top of regular academic stress (e.g., tests, assignments, busy schedules) may be one explanation for poorer academic outcomes seen in Black students, these associations have been incompletely examined and are poorly understood [16].

There are several theoretical and contextual models that have been proposed to explain the relationship between perceived racism and discrimination and negative health outcomes. Clark and colleagues proposed a biopsychosocial model to better understand racism as a stressor that may lead to negative health outcomes [17]. In their model, an environmental stimulus (e.g., interpersonal interaction) can be perceived as racist. In turn, this perception of racism and discrimination (described as a stressor) can lead to psychological and physiological stress responses and subsequently negative health outcomes. There are also potential moderators and mediators in this contextual model. The authors describe constitutional factors, sociodemographic factors, and psychological/behavioral factors that may moderate the relationship between the environmental stimuli and the perception of racism. Additionally, coping response, both adaptive (e.g., exercising, meditating) and maladaptive (e.g., substance use), may mediate the relationship between the perception of discrimination and psychological and physiological stress responses [18]. One of the coping strategies that may attenuate the relationship between stress (e.g., perceived discrimination) and negative health outcomes (e.g., depression and anxiety) is social support.

Perceived social support may mitigate the effect of stressors on negative health outcomes [19-22]. Conversely, the lack of a strong social support system and connectedness has also been linked to symptoms of depression and anxiety [22]. Given the demands of medical school (heavy course load, standardized exams), students often report social isolation, which may help explain the higher rates of mental health symptoms among medical students. There are racial and gender differences in social support among medical students [7]. For example, Hardeman and colleagues found that Black medical students were at an $83 \%$ greater risk of lacking social support compared to their White counterparts; females were $24 \%$ more likely to report social support compared to males [7].

Using Clark and colleagues' biopsychosocial model, the current study examines the association between socialinterpersonal experiences (e.g., environmental stimulus) that may be perceived as discriminatory (stressor), social support (coping strategy/response), and symptoms of depression and anxiety (negative health outcomes) among Black medical students (see Fig. 1) [17]. Given the differences in the prevalence of social support and connectedness as well as mental health symptoms by gender, the study will also examine differences in the association between perceived discrimination, connectedness, and mental health symptoms by gender.

\section{Methods}

\section{Overview}

The study utilizes data collected from a convenience sample of Black medical students via an anonymous 37-item electronic questionnaire using Qualtrics software (see Appendix). The survey was open for 2 weeks in August 2020. The survey included demographics, peer connectedness, experiences of discrimination, and symptoms of depression and anxiety. The study was determined to be exempt by the Institutional Review Board at Michigan State University.

\section{Procedures}

The electronic survey was distributed through the Student National Medical Association (SNMA) listserv. The SNMA was chosen due to its role as a governing body for underrepresented minority medical students. SNMA facilitated the survey distribution through their weekly digest listserv $[23,24]$. In addition, the survey was distributed to leaders of the Organization of Student Representatives (OSR) and to the Association of American Medical College (AAMC). The OSR is an auxiliary branch of the AAMC that serves to 


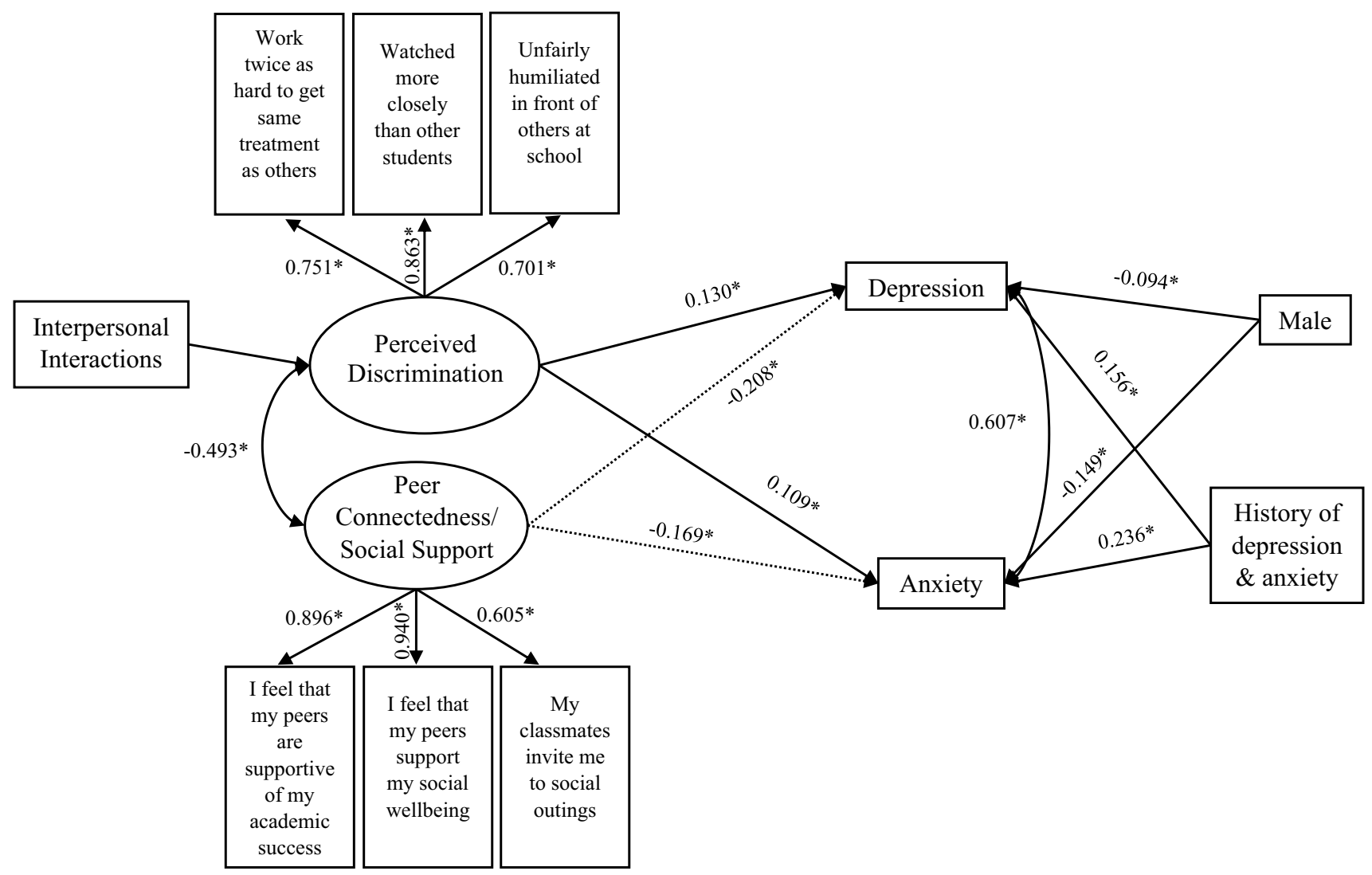

Fig. 1 Structural equation model: experiences of discrimination, peer connectedness, and mental health symptoms among Black medical students

incorporate students into the governance of the AAMC and facilitate student action on healthcare issues. Finally, posts on social media accounts contained a link to the survey, requesting that only current medical students complete the survey. The recruitment letter invited students to complete a short survey regarding the medical school experience of self-identified African American medical students. Students were advised that participation in the survey was voluntary and completed a consent form at the start of the survey. All participants were eligible to receive a $\$ 10$ Amazon gift card upon completion of the survey.

\section{Measures}

Mental Health Outcomes The Patient-Reported Outcomes Measurement Information System (PROMIS) Depression Short Form 8a and Anxiety Short Form 8a were used to measure symptoms of depression and anxiety. The depression form includes eight statements scored on a 5-point Likert scale (never, rarely, sometimes, often, always) that assess symptoms in the past 7 days (e.g., In the past 7 days, I felt hopeless). Similarly, the anxiety form includes eight statements assessing the past 7 days (e.g., In the past 7 days, I felt uneasy), answered on the same Likert scale. The total possible range for the raw depression and anxiety scale scores is 11 to 55 . The raw scores were transformed to T-scores created based on the US general population with a mean of 50 and a standard deviation of 10. Both PROMIS scales have been validated for use with adults and children and have acceptable validity and reliability [25]. Higher T-scores indicate more symptoms of depression or anxiety. Cronbach's alphas were 0.93 for both the depression and anxiety symptom scales for the current study.

Experiences of Discrimination We used the abbreviated version of the Chronic Work Discrimination survey created for the Chicago Community Adult Health Study (CCAHS) to assess discrimination experiences [26]. This work discrimination scale for the CCAHS was adapted from the Perceived Racism Scale [27]. The survey includes 3 items scored (e.g., How often are you unfairly humiliated in front of others at school?) on a 5-point Likert scale (never, less than once a year, a few times a year, a few times a month, once a week or more). Cronbach's alpha for this scale in the current study was 0.78 . 
Peer Connectedness Three items were used to measure peer connectedness. Items included (1) I feel that my peers are supportive of my academic success, (2) I feel that my peers support my social wellbeing, and (3) My classmates invite me to social outings. These items were scored on a 5-point Likert scale (never, rarely, sometimes, often, always). The total possible score for peer connectedness and characteristics ranges from 3 to 15 . Cronbach's alpha for this scale in the current study was 0.83 .

\section{Statistical Analyses}

Descriptive statistics were assessed; frequencies were used for categorical variables, mean and standard deviations were used for continuous variables. Path models in Mplus 7.3 were used to examine the relationship between experiences of discrimination, peer connectedness, and symptoms of depression and anxiety among Black medical students. The models adjusted for year in medical school, ethnicity, gender, and diagnosis of depression or anxiety prior to medical school. Year in medical school was selected to control for possible differences in discrimination/racism as students transition from course work (1st and 2 nd year) to clinical duties in the hospital (3rd and 4th year). Year in medical school was correlated with age; therefore, age was not included in the analyses. The root mean square error of approximation (RMSEA), comparative fit index (CFI), and Tucker-Lewis index (TLI) were used to assess model fit. RMSEA values $\leq 0.05$, CFI values $\geq 0.95$, and TLI values $\geq 0.90$ generally represent a good fit to the observed data.
There were 750 students who completed the survey; 733 (97.7\%) reported they were Black. Of the 733 Black students who completed the survey, $93.3 \%(n=684)$ had no missing data. There were no differences in the Black participants with no missing data $(n=684)$ compared to Black participants with missing data $(n=49)$ in terms of demographics or depression/anxiety T-scores $(p>0.05)$; data were deemed to be missing at random. Mplus uses full information maximum likelihood to compute parameter estimates for cases with missingness (assuming missing at random) using all available data.

\section{Results}

Table 1 displays demographics as well as connectedness, experiences of discrimination, and mental health symptoms for the entire sample and stratified by gender. The sample was $80 \%$ female $(n=569)$, the average age was 25.6 $(\mathrm{SD}=3.4), 43.4 \%$ were in their third or fourth year of medical school, and $13 \%$ had a clinical diagnosis of depression or anxiety before medical school $(n=92)$. Approximately half of the students reported being watched more closely than other students a few times a month or more $(n=358,50.5 \%)$, and $66 \%$ reported they felt they had to work twice as hard as others to get the same treatment or evaluation $(n=468)$; there were no differences by gender $(p>0.050)$. Most students reported that their peers were supportive of their academic success often or always $(n=430,60.7 \%)$, and 53\% reported that students often or always invited them to social

Table 1 Experiences of discrimination, peer connectedness, and mental health symptoms among Black medical students

\begin{tabular}{|c|c|c|c|c|}
\hline & $\begin{array}{l}\text { Pooled sample } \\
n(\%)\end{array}$ & Females & Males & $p$ \\
\hline Male & $139(19.6)$ & - & - & \\
\hline Year in medical school & & & & .704 \\
\hline $1 \mathrm{st}$ & $216(30.6)$ & $167(29.4)$ & $49(35.3)$ & \\
\hline 2nd & $184(26.0)$ & $151(26.6)$ & $33(23.7)$ & \\
\hline $3 \mathrm{rd}$ & $158(22.3)$ & $126(22.2)$ & $32(23.0)$ & \\
\hline 4 or more & $149(21.1)$ & $124(21.8)$ & $25(18.0)$ & \\
\hline \multicolumn{5}{|l|}{ Discrimination (few times a month or more) } \\
\hline $\begin{array}{l}\text { How often do you feel that you have to work twice as hard as others to } \\
\text { get the same treatment or evaluation? }\end{array}$ & $468(66.1)$ & $378(66.9)$ & $90(64.7)$ & .947 \\
\hline How often are you watched more closely than other students? & $358(50.5)$ & $288(51.2)$ & $70(50.8)$ & .451 \\
\hline How often are you unfairly humiliated in front of others at school? & $60(8.4)$ & $49(8.5)$ & $12(8.7)$ & .465 \\
\hline \multicolumn{5}{|l|}{ Peer connectedness (often/always) } \\
\hline I feel that my peers are supportive of my academic success & $430(60.7)$ & $330(58.3)$ & $100(71.9)$ & .006 \\
\hline I feel that my peers support my social wellbeing & $400(56.5)$ & $304(53.7)$ & $96(69.0)$ & .006 \\
\hline My classmates invite me to social outings & $374(52.9)$ & $284(50.2)$ & $90(64.7)$ & .015 \\
\hline Clinical diagnosis of depression or anxiety before medical school & $92(13.0)$ & $74(13.0)$ & $18(12.9)$ & .986 \\
\hline Mean depression $T$-score (SD) & $53.6(7.8)$ & $54.0(7.8)$ & $52.1(7.9)$ & .011 \\
\hline Mean anxiety $T$-score (SD) & $58.6(8.4)$ & $59.2(8.2)$ & $56.0(8.4)$ & $<.001$ \\
\hline
\end{tabular}


outings $(n=374)$; males reported more connectedness compared to females on all three variables $(p<0.050)$. The mean depression T-score for the entire sample was 53.6 $(\mathrm{SD}=7.8)$, and the mean anxiety $\mathrm{T}$-score was $58.6(\mathrm{SD}=8.4)$.

Structural equation modeling was used to assess the association between experiences of discrimination, peer connectedness, and mental health symptoms; the fit indices were acceptable $(\mathrm{CFI} / \mathrm{TFI}=0.982 / 0.973$; $\mathrm{RMSEA}=0.055)$, and standardized estimates are presented (see Table 2 and Fig. 1). The T-score for anxiety and depression was lower among males (estimate $=-0.094, p=0.011$ and estimate $=-0.149, p<0.001$ for depression and anxiety, respectively). The students' year in medical school was not associated with depression nor anxiety T-scores. A history of depression and/or anxiety prior to medical school was positively associated with both symptoms of depression (estimate $=0.156, p<0.001$ ) and anxiety (estimate $=0.236$, $p<0.001)$. Students' connectedness to peers was associated with lower T-scores for depression (estimate $=0.208$, $p<0.001$ ) and anxiety symptoms (estimate $=-0.169$, $p<0.001)$. Experiences of discrimination were associated with more symptoms of depression (estimate $=0.130$, $p=0.006$ ) and anxiety (estimate $=0.109, p=.020$ ). There was a strong negative association between connectedness and experiences of discrimination such that more connectedness was associated with fewer experiences of discrimination (estimate $=-0.493, p<0.001$ ). There was also a strong positive association between anxiety and depression $\mathrm{T}$-scores (estimate $=0.607, p<0.001$ ).

Given differences in depression and anxiety symptoms by gender, we stratified the analyses by gender. Among females $(\mathrm{CFI} / \mathrm{TFI}=0.987 / 0.981 ; \mathrm{RMSEA}=0.050)$, having a clinical diagnosis of depression or anxiety prior to medical school was associated with higher T-scores for depression and anxiety symptoms (estimate $=0.150, p<0.001$, and estimate $=0.243, p<0.001$, respectively). Peer connectedness was negatively associated with depression and anxiety symptoms such that female students who felt more connected had lower T-scores (estimate $=-0.237, p<0.001$ and estimate $=-0.164, p<0.001$, respectively). Experiences of discrimination were not associated with either symptoms of depression or anxiety and females. Among males $(\mathrm{CFI} / \mathrm{TFI}=0.988 / 0.982 ; \mathrm{RMSEA}=0.046)$, a clinical diagnosis of depression or anxiety prior to medical school was associated with depression but not anxiety symptoms (estimate $=0.185, p=0.048$ and estimate $=0.228, p=0.068$, respectively). There was a negative association between peer connectedness and anxiety symptoms (estimate $=-0.215$, $p=0.002$ ), but there was no association between connectedness and depression symptoms (estimate $=-0.148$, $p=0.153$ ). Experiences of discrimination were associated with higher depression and anxiety $\mathrm{T}$-scores such that more frequent experiences of discrimination were associated with more symptoms of depression and anxiety (estimate $=0.309$, $p=0.001$, and estimate $=0.244, p=0.003$ ).

\section{Discussion}

This investigation used a previously established conceptual framework to explore the association between peer connectedness, experiences of discrimination, and mental health
Table 2 Standardized estimates: experiences of discrimination, peer connectedness, and mental health symptoms among Black medical students

\begin{tabular}{lllll}
\hline Two-tailed & & & & \\
\hline & Estimate & S.E & Est./S.E & $p$-value \\
\hline Depression on & & & & \\
$\quad$ Male & -.094 & .037 & -2.547 & .011 \\
Year in medical school & -.004 & .448 & -.009 & .993 \\
Ethnicity & -.024 & .041 & -.583 & .560 \\
History of depression and/or anxiety prior to medical school & .156 & .034 & 4.571 & $<.001$ \\
Peer connectedness & -.208 & .045 & -4.652 & $<.001$ \\
Racism/discrimination & .130 & .047 & 2.745 & .006 \\
Anxiety on & & & & \\
Male & -.149 & .036 & -4.111 & $<.001$ \\
Year in medical school & -.018 & .688 & -.026 & .980 \\
Ethnicity & -.072 & .033 & -2.393 & .028 \\
History of depression and/or anxiety prior to medical school & .236 & .042 & 5.658 & $<.001$ \\
Peer connectedness & -.169 & .043 & -3.921 & $<.001$ \\
Racism/discrimination & .109 & .047 & 2.318 & .020 \\
Racism/Discrimination with Peer Connectedness & -.493 & .035 & -14.201 & $<.001$ \\
Anxiety with depression & .607 & .020 & 30.421 & $<.001$ \\
\hline
\end{tabular}

$\mathrm{CFI} / \mathrm{TFI}=0.982 / 0.973 ; \mathrm{RMSEA}=0.055$ 
symptoms among Black medical students. We also explored if gender moderated these relationships. Consistent with previous findings, the medical students in our sample reported more symptoms of depression and anxiety given T-scores in our sample were higher than the general population (mean depression and anxiety T-scores of 53.6 and 58.6 compared to 50.0 for the general population). The mean anxiety T-score in our study (58.6) was similar to previous studies among medical students [7]. Consistent with Clark and colleagues' biopsychosocial model, we also identified a positive association between environmental stimuli that may be perceived as discriminatory (e.g., you have to work twice as hard as others to get the same treatment or evaluation) and mental health symptoms among males such that more experiences of discrimination were associated with more symptoms of depression and anxiety among Black male medical students [17]. Despite the well-documented negative effects of discrimination on student well-being and performance, opportunities for improvement are abundant. Our study found that peer connectedness, a potential coping strategy, was associated with fewer symptoms of depression among males and females and fewer symptoms of anxiety among females. The protective effect of connectedness on symptoms of depression and anxiety may suggest that interventions that target this area, like faculty mentorship, peer, and near-peer engagement, may represent potential targetable strategies to improve the wellness of Black medical students.

\section{Gender Differences}

There are established gender differences in connectedness and social support as well as mental health outcomes. Similar to previous studies, female medical students in the current study reported more symptoms of depression and anxiety [1,7], unlike previous studies; however, males in our study reported higher rates of peer connectedness [7]. This may speak to the possibility that the socially distant environment cultivated by the response to COVID-19 had a different impact on opportunities for connectedness and social support for each gender. Connectedness was protective for both symptoms of depression and anxiety among females but only anxiety symptoms among males. There were no differences in the frequency of experiences of discrimination by gender. Experiences of discrimination were associated with symptoms of depression and anxiety among males with no significant relationship among females. This is consistent with current literature suggesting that gender has been found to moderate the relationship between discrimination and depression [28, 29]. Studies have found that increases in perceived discrimination during adolescence predicted an increase in symptoms of depression and anxiety during early adulthood among Black males but not females [27]. Similarly, another study found that perceived discrimination during adolescence was associated with later substance abuse and poorer eating habits among males, while there was no relationship among females [28]. This highlights the concept of intersectionality which explains how certain demographics (or characteristics) can compound themselves and increase risk [29]; the intersection of Black race, male gender, being a medical student, and perceptions of external stimuli as discriminatory (e.g., interpersonal interactions perceived as discriminatory) may compound themselves to predict symptoms of depression and anxiety. Similarly, the intersection of Black race, female gender, and being a medical student may compound and synergistically increase the risk of depression and anxiety symptoms (as female students had more symptoms of depression and anxiety compared to males).

Using the biopsychosocial model by Clark and colleagues, we examined social-interpersonal interactions that may be perceived as discriminatory in the context of medical education while identifying potential coping strategies to mitigate these harmful effects (i.e., peer connectedness) [17]. Collectively, our results suggest that efforts to potentiate the success and wellness of a diverse student body should endeavor to robustly address issues of discrimination and social isolation. Moreover, these results point to the urgent need for an intersectional approach to disparities within undergraduate medical education.

\section{Limitations}

This study had a few limitations that should be discussed. First, a convenience sample was used, which limits generalizability. Relatedly, the use of social media and email listservs limits our ability to report a response rate, and we were unable to provide the distribution of participants from each source. Of note, our sample demographics and the prevalence of depressive symptoms was similar to other studies of US medical students [7]. Second, the study utilized a cross-sectional design, so temporal relationships among the main variables of connectedness, mental health, and discrimination cannot be assessed. Future studies should examine these relationships over time and include additional outcomes (e.g., academic achievement, matching into residency, achieving board certification). There are also potential cofounders that were not included in the study including socioeconomic status and marital status. Lastly, we did not assess whether medical students attributed their experiences of discrimination to race. Although Black persons attribute the vast majority of discrimination to race and ethnicity, experiences of discrimination could stem from other demographics and identities (e.g., age, weight, sexual orientation) [30]. Despite these limitations, this is one of 
only a few studies that has specifically looked at mental health outcomes among Black medical students.

As institutions work to address the physician shortage and lack of diversity in the physician workforce, we must first examine and address issues of discrimination within medical training. Every year, Black students matriculate into medical schools nationwide and subsequently experience discrimination that may hinder their success. Our results raise concerns regarding the systems in place to combat discrimination of Black medical students, systemic racism within the healthcare field they are training to enter, and resources available for students dealing with poor mental health. It is imperative that institutions create a system that allows students to feel safe reporting and addressing discrimination issues. Institutions should also investigate practices that can be implemented to increase Black medical students' feelings of support and connectedness, as this is seen to lead to fewer reports of discrimination.

Supplementary Information The online version contains supplementary material available at https://doi.org/10.1007/s40615-021-01157-7.

Author Contribution All authors contributed to the manuscript as follows: A.J. Milam created the design of the article, led the study, and assisted with writing; $\mathrm{O}$. Oboh and $\mathrm{Z}$. Brown drafted the article section; J. Edwards-Jonson conducted the data analysis and assisted with writing; A. Terry created the survey questions used for data collection and assisted with writing; C. Barajas assisted with writing as well as the survey and article format; K. Simon and C. D. M. Furr-Holden assisted with writing and completed a final review of the article. All authors approved the final version of the article.

Funding Support for this work came from the National Institute on Minority Health and Health Disparities of the National Institutes of Health (U54MD011227). National Institute on Drug Abuse K12DA000357.

Availability of Data and Material Available upon request.

Code Availability Mplus v7.3

\section{Declarations}

Ethics Approval The study was determined to be exempt by the Institutional Review Board at Michigan State University.

Consent to Participate Participants provided consent.

Consent for Publication Not applicable.

Conflict of Interest The authors declare no competing interests.

\section{References}

1. Dyrbye LN, Thomas MR, Shanafelt TD. Systematic review of depression, anxiety, and other indicators of psychological distress among US and Canadian medical students. Acad Med. 2006;81(4):354-73.

2. Dutheil F, Aubert C, Pereira B, Dambrun M, Moustafa F, Mermillod M, Navel V. Suicide among physicians and health-care workers: a systematic review and meta-analysis. PloS One. 2019;14(12):e0226361.

3. Dyrbye LN, West CP, Satele D, et al. Burnout among US medical students, residents, and early career physicians relative to the general US population. Acad Med. 2014;89(3):443-51.

4. Kumar S. Burnout and doctors: prevalence, prevention and intervention. Healthcare (Basel). 2016;4(3):37.

5. Dyrbye LN, Thomas MR, Massie FS, et al. Burnout and suicidal ideation among US medical students. Ann Intern Med. 2008;149(5):334-41.

6. Rotenstein LS, Ramos MA, Torre M, et al. Prevalence of depression, depressive symptoms, and suicidal ideation among medical students: a systematic review and meta-analysis. JAMA. 2016;316(21):2214-36. https://doi.org/10.1001/jama.2016.17324.

7. Hardeman RR, Przedworski JM, Burke SE, et al. Mental wellbeing in first year medical students: a comparison by race and gender. J Racial Ethn Health Disparities. 2015;2(3):403-13.

8. Salk RH, Hyde JS, Abramson LY. Gender differences in depression in representative national samples: meta-analyses of diagnoses and symptoms. Psychol Bull. 2017;143(8):783-822. https:// doi.org/10.1037/bul0000102.

9. Depression Risk Factors. National Institute of Mental Health. Published 2018. Accessed May 14, 2018. https://www.nimh.nih. gov/health/topics/depression/index.shtml

10. Stoolmiller M, Kim HK, Capaldi DM. The course of depressive symptoms in men from early adolescence to young adulthood: identifying latent trajectories and early predictors. J Abnorm Psychol. 2005;114(3):331-45.

11. McKercher C, Sanderson K, Schmidt MD, Otahal P, Patton GC, Dwyer T, Venn AJ. Physical activity patterns and risk of depression in young adulthood: a 20-year cohort study since childhood. Soc Psychiatry Psychiatr Epidemiol. 2014;49(11):1823-34.

12. Dyrbye L, Shanafelt T. A narrative review on burnout experienced by medical students and residents. Med Educ. 2016;50(1):132-49. https://doi.org/10.1111/medu.12927.

13. Dyrbye LN, Massie FS, Eacker A, et al. Relationship between burnout and professional conduct and attitudes among US medical students. JAMA. 2010;304(11):1173-80. https://doi.org/10.1001/ jama.2010.1318.

14. Pascoe EA, Smart RL. Perceived discrimination and health: a meta-analytic review. Psychol Bull. 2009;135(4):531-54. https:// doi.org/10.1037/a0016059.

15. Smedley BD, Stith AY, Nelson AR, eds. Unequal treatment: confronting racial and ethnic disparities in health care. NAP (US). 2003;17.

16. Greer TM, Chwalisz K. Minority-related stressors and coping processes among African American college students. J Coll Stud Dev. 2007;48(4):388-404.

17. Clark R, Anderson NB, Clark VR, Williams DR. Racism as a stressor for African Americans: a biopsychosocial model. Am Psychol. 1999;54(10):805.

18. Linnabery E, Stuhlmacher AF, Towler A. From whence cometh their strength: social support, coping, and well-being of Black women professionals. Cultur Divers Ethnic Minor Psychol. 2014;20(4):541-9.

19 Shimanoe C, Hara M, Nishida Y, Nanri H, Otsuka Y, Horita M, Tanaka K. Coping strategy and social support modify the association between perceived stress and C-reactive protein: a longitudinal study of healthy men and women. Stress. 2018;21(3):237-46.

20. Griffin EK, Armstead C. Black's coping responses to racial stress. J Racial Ethn Health Disparities. 2020;7(4):609-18.

21. Lemon ED, Vu M, Roche KM, Hall KS, Berg CJ. Depressive symptoms in relation to adverse childhood experiences, 
discrimination, hope, and social support in a diverse sample of college students. J Racial Ethn Health Disparities. 2021;1-11.

22. Thompson G, McBride RB, Hosford CC, Halaas G. Resilience among medical students: the role of coping style and social support. Teach Learn Med. 2016;28(2):174-82.

23. Chisholm LP, Jackson KR, Davidson HA, Churchwell AL, Fleming AE, Drolet BC. Evaluation of racial microaggressions experienced during medical school training and the effect on medical student education and burnout: a validation study. J Natl Med Assoc. 2021;113(3):310-4.

24. Anderson, N, Lett, E, Asabor, EN, et al. The association of microaggressions with depressive symptoms and institutional satisfaction among a national cohort of medical students. J Gen Intern Med. 2021;1-10.

25. Office of Strategic Coordination - The Common Fund. Programs Snapshot. National Institutes of Health. Published 2018. Accessed December 13, 2018. https://commonfund.nih.gov/promis/index

26. McNeilly MD, Anderson NB, Armstead CA, et al. The perceived racism scale: a multidimensional assessment of the experience of white racism among African Americans. Ethn Dis. 1996;6(1-2):154-66.
27. Assari S, Moazen-Zadeh E, Caldwell CH, Zimmerman MA. Racial discrimination during adolescence predicts mental health deterioration in adulthood: gender differences among Blacks. Front Public Health. 2017;5:104. https://doi.org/10.3389/fpubh. 2017.00104.

28. Brodish AB, Cogburn CD, Fuller-Rowell TE, Peck S, Malanchuk O, Eccles JS. Perceived racial discrimination as a predictor of health behaviors: the moderating role of gender. Race Soc Probl. 2011;3(3):160-9. https://doi.org/10.1007/s12552-011-9050-6.

29. Evans CR, Erickson N. Intersectionality and depression in adolescence and early adulthood: a MAIHDA analysis of the national longitudinal study of adolescent to adult health, 1995-2008. Soc Sci \& Med. 2019;220:1-11.

30. Kessler RC, Mickelson KD, Williams DR. The prevalence, distribution, and mental health correlates of perceived discrimination in the United States. J Health Soc Behav. 1999;1999(4):208-30.

Publisher's Note Springer Nature remains neutral with regard to jurisdictional claims in published maps and institutional affiliations. 